\title{
Reconstruction of the right ventricular outflow tract with a bovine jugular vein graft fixed with a naturally occurring crosslinking agent (genipin) in a canine model
}

\author{
Yen Chang, MDa \\ Chen-Chi Tsai, $\mathrm{PhD}^{\mathrm{b}}$ \\ Huang-Chien Liang, MS ${ }^{\mathrm{C}}$ \\ Hsing-Wen Sung, $\mathrm{PhD}^{\mathrm{C}}$
}

From the Division of Cardiovascular Surgery, a Veterans General Hospital-Taichung, and College of Medicine, National Yang-Ming University, Taipei, Taiwan; Biomedical Engineering Center, ${ }^{\mathrm{b}}$ Industrial Technology Research Institute, Hsinchu, Taiwan; and the Department of Chemical Engineering, ${ }^{c}$ National Tsing Hua University, Hsinchu, Taiwan.

This work was supported by a grant from the National Science Council of Taiwan, Republic of China (NSC-88-2314-E-008002).

Received for publication Feb 14, 2001; revisions requested March 20, 2001; revisions received April 10, 2001; accepted for publication April 21, 2001

Address for reprints: Hsing-Wen Sung, $\mathrm{PhD}$, Department of Chemical Engineering, National Tsing Hua University, Hsinchu, Taiwan 30013 (E-mail: hwsung@ che.nthu. edu.tw).

J Thorac Cardiovasc Surg 2001;122:1208-18

Copyright (C) 2001 by The American Association for Thoracic Surgery

0022-5223/2001 \$35.00 + $0 \quad \mathbf{1 2 / 1 / 1 1 7 6 2 4}$

doi:10.1067/mtc.2001.117624
Objective: This study was designed to evaluate a newly developed biologic valved conduit fixed with genipin used to reconstruct the right ventricular outflow tract in a canine model.

Methods: Fresh bovine jugular veins with a retained native valve procured from a slaughterhouse were used as raw materials to fabricate the valved conduits. A naturally occurring crosslinking agent, genipin, was used to fix the procured jugular veins. The glutaraldehyde-fixed counterpart was used as a control. A canine model was used in the study.

Results: Echocardiography revealed that the motion of the valvular leaflets in both the glutaraldehyde- and genipin-fixed conduits was satisfactory. The transvalvular pressure gradients of both studied groups were minimal. No endothelium-like cells were observed on the luminal surface of the conduit and the valvular leaflet for the glutaraldehyde-fixed group throughout the entire course of the study. In contrast, endothelium-like cells were observed on the entire surface of the genipin-fixed valved conduit retrieved at 6 months postoperatively in all the cases studied. There was no evidence of luminal fibrous peel in any the valved conduits studied. Degradation of valvular leaflet in one of the glutaraldehyde-fixed conduits was observed. In this particular case, thrombus formation was also observed on the surface of the valvular leaflet. On the other hand, no apparent degradation or thrombus formation was observed on the surfaces of the genipin-fixed valvular leaflet and conduit. A significantly more severe inflammatory reaction was observed for the glutaraldehyde-fixed conduit than for its genipin-fixed counterpart throughout the entire course of the study. The calcium contents of the samples before implantation and those retrieved at distinct implantation duration were minimal for both the glutaraldehyde- and genipin-fixed tissues.

Conclusion: Although further studies are necessary, the genipin-fixed valved conduit appears to have great potential in helping mitigate the complications observed in the commercially available conduits.

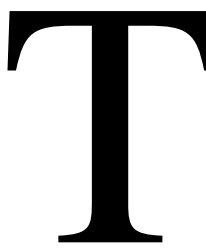

here is a great medical need for the use of external valved conduits in the repair of many congenital cardiac anomalies to reconstruct the right ventricular outflow tract (RVOT) ${ }^{1-9}$ Currently available valved conduits can be divided into 2 primary categories: allograft and xenograft. For the reconstruction of the RVOT, Homann and associates ${ }^{9}$ reported that allografts show significantly better longterm durability than xenografts, regardless of the age at implantation and the diameter of the valved conduit. Although allografts are considered the conduit of 
choice, their availability is limited, and therefore xenografts are implanted as well. ${ }^{9}$

The xenograft valved conduits currently available consist of a synthetic Dacron graft with a tissue or mechanical valve. Despite many endeavors in past decades, the clinical results of RVOT reconstruction with these xenograft valved conduits are still not satisfactory. Specific problems encountered clinically include suboptimal hemodynamic performance and conduit kinking or compression. ${ }^{5-7}$ In addition, fibrous peeling from the Dacron graft, which eventually results in valve dysfunction, has been reported previously. 5,7

To address these deficiencies, we undertook the development of a new biologic valved conduit: a bovine jugular vein graft with a retained native valve crosslinked with a naturally occurring crosslinking agent, genipin. The retained native valve in the bovine jugular vein used in the study had 2 leaflets. Trileaflet valvular configuration can also be obtained in these natural conduits.

Clinically, the most commonly used crosslinking agent for biologic tissue fixation is glutaraldehyde. ${ }^{10}$ However, concerns exist regarding calcification, cytotoxicity, and long-term durability with the currently available glutaraldehyde-fixed bioprostheses. ${ }^{11-14}$ In an attempt to overcome these problems, our group has used a naturally occurring crosslinking agent, genipin, to fix biologic tissues. ${ }^{15-18}$

Genipin and its related iridoid glucosides extracted from the fruits of Gardenia jasminoides Ellis have been widely used as an antiphlogistics and cholagogues in herbal medicine. ${ }^{19}$ Additionally, genipin can spontaneously react with amino acids or proteins to form dark-blue pigments. ${ }^{20,21}$ These dark-blue pigments have been used in the fabrication of food dyes. The cytotoxicity of genipin was previously studied by our group in vitro with $3 \mathrm{~T} 3$ fibroblasts. ${ }^{17}$ Glutaraldehyde was used as a control. The results implied that genipin is significantly less cytotoxic than glutaraldehyde. Additionally, the genotoxicity of genipin was tested in vitro with Chinese hamster ovary (CHO-K1) cells. ${ }^{18}$ The results hinted that glutaraldehyde may produce a weakly clastogenic response in $\mathrm{CHO}-\mathrm{K} 1$ cells. In contrast, genipin does not cause a clastogenic response in $\mathrm{CHO}-\mathrm{K} 1$ cells.

The biocompatibility of the genipin-fixed tissue was studied in a growing rat model subcutaneously. ${ }^{16}$ It was noted that the inflammatory reaction of the genipin-fixed tissue was significantly less than that of its glutaraldehydefixed counterpart. Furthermore, the calcium content of the genipin-fixed tissue measured throughout the entire course of the study was minimal. This implied that genipin may form biocompatible crosslinked products. The aforementioned results indicated that genipin is an effective crosslinking agent for biologic tissue fixation.

This study was designed to evaluate the performance of this newly developed biologic valved conduit fixed with genipin to reconstruct the RVOT in a canine model. The glutaraldehyde-fixed counterpart was used as a control.

\section{Materials and Methods Preparation of the Valved Conduits}

Fresh bovine jugular veins (approximately 14-16 mm) with a retained native valve procured from a slaughterhouse were used as raw materials to fabricate the valved conduits tested in the study. The procured bovine jugular veins were transported in a cold normal saline solution. On return, the jugular veins first were gently rinsed with fresh saline solution to remove excess blood on the tissue. Adherent fat was then carefully trimmed from the tissue surface. Subsequently, a column of saline solution in a pressure of approximately $10 \mathrm{~mm} \mathrm{Hg}$ was filled into the jugular vein from its distal end to check the valve competency. Only those jugular veins with a competent valve were used in the study.

The jugular veins then were mounted onto a fixation tank. The fixation tank was filled with a $0.625 \%$ aqueous glutaraldehyde or a $0.625 \%$ aqueous genipin (Challenge Bioproducts Co, Taichung, Taiwan) solution buffered with phosphate-buffered saline solution (pH 7.4) at room temperature. The mounted vein was fixed with its valve closed by applying a $10-\mathrm{mm} \mathrm{Hg}$ column of fixation solution into the conduit from its distal end. It was found in our study that the retained valves in the bovine jugular veins were at times incompetent when a zero or minimal pressure $(2-3 \mathrm{~mm} \mathrm{Hg})$ was applied on the valves during fixation. A $10-\mathrm{mm} \mathrm{Hg}$ pressure was therefore applied on the valves during fixation to ensure that the retained valves in the bovine jugular veins were competent in a physiologic environment. The fixation solution was circulated through the vein through its proximal end for about 15 minutes every 3 to 4 hours. The fixation process was accomplished as per this procedure repetitively for 3 days. The maximum time period between retrieval and initiation of tissue fixation was less than 6 hours.

The fixed valved conduits were sterilized in a graded series of ethanol solutions, with a gradual increase in concentration from $20 \%$ to $75 \%$ over a period of 4 hours. Finally, the conduits were rinsed in sterilized phosphate-buffered saline solution and prepared for the animal study.

\section{Implantation of the Valved Conduits}

Sixteen mongrel dogs, each weighing about 20 to $25 \mathrm{~kg}$, were used in the study to evaluate the performance of the valved conduits prepared. A left thoracotomy was performed through the fourth intercostal space after achievement of general anesthesia. An initial dose of $100 \mathrm{U} / \mathrm{kg}$ body weight of heparin was administered. The RVOT was partially clamped longitudinally, and an incision was made. The proximal side-to-end anastomosis from the RVOT to the proximal end of the conduit was completed with 5-0 polypropylene sutures by using a continuous suturing technique. Then the proximal portion of the conduit was clamped, and the clamp for the RVOT was released. After the main pulmonary artery (PA) was partially clamped, suturing of the end-to-side distal anastomosis was made from the conduit to the PA with continuous 6-0 polypropylene sutures. All clamps were released, and the main PA was ligated between the anastomoses of the bypass conduit and distally to the native pulmonic valve. No antithrombotic drugs were administered to the dogs after the operation. Animal care and use was performed in compliance 

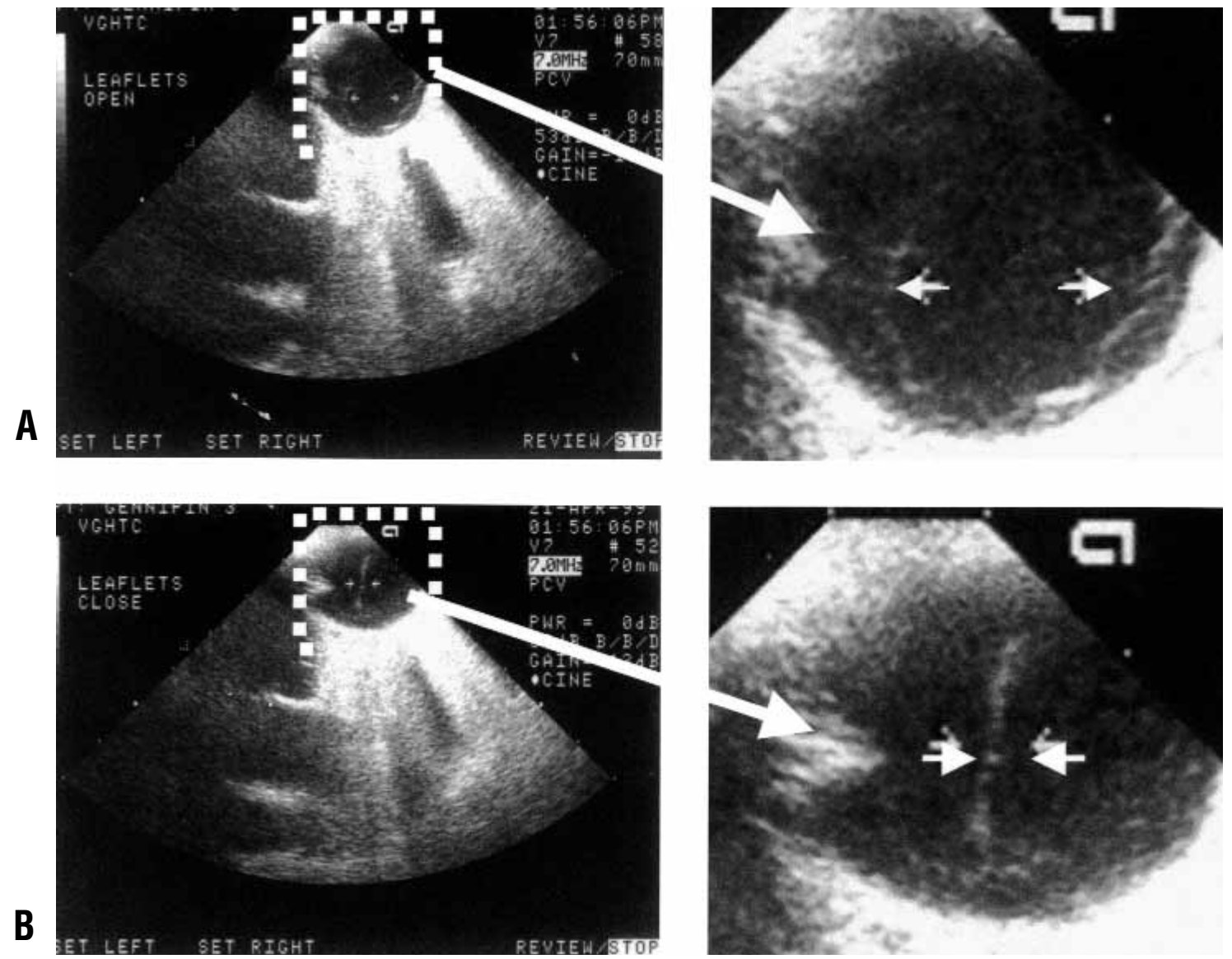

Figure 1. Two-dimensional echocardiographic images of the valvular leaflet motion of a genipin-fixed valved conduit obtained at 6 months postoperatively: A, During opening; B, at closure.

with the "Guide for the Care and Use of Laboratory Animals" prepared by the Institute of Laboratory Animal Resources, National Research Council, and published by the National Academy Press, revised 1996.

\section{Echocardiographic Examination}

The functional and hemodynamic performance of the implanted valved conduits was examined with an echocardiographic system (HP Sonos 5500; Hewlett-Packard Company, Palo Alto, Calif). Two-dimensional echocardiographic examination of the motion of the valvular leaflets was conducted directly on the conduits immediately after implantation and at retrieval with the animal during general anesthesia. The peak transvalvular pressure gradient was calculated from a simplified Bernoulli equation. ${ }^{22}$

\section{Retrieval of the Implanted Valved Conduits}

The implanted valved conduits were retrieved at 1 month $(n=4$ for each studied group) and 6 months ( $n=4$ for each studied group) postoperatively. Before retrieval, the animals were given heparin. At retrieval, the appearance of each retrieved valved conduit was grossly examined. The retrieved conduits then were processed for light microscopy for histologic examination or atomic absorption analysis to determine calcium content.

\section{Light Microscopy}

All retrieved conduits were rinsed in physiologic saline solution to remove excess blood. The samples used for light microscopy were fixed in $10 \%$ phosphate-buffered formalin for at least 3 days and prepared for histologic examination. In the histologic examination the fixed samples were embedded in paraffin and sectioned into a thickness of $5 \mu \mathrm{m}$ and then stained with hematoxylin and eosin or von Kossa stain. The stained sections of each test sample then were examined with light microscopy (Nikon Microphoto-FXA) for tissue inflammatory reaction (hematoxylin and eosin) and photographed with a 100 ASA Kodachrome film.

\section{Atomic Absorption Analysis}

The atomic absorption analysis was used to determine the calcium content of each retrieved sample. In the analysis the retrieved samples of each studied group first were lyophilized for 24 hours and weighed. The lyophilized sample then was immersed in a $6 \mathrm{~N} \mathrm{HCl}$ solution (approximately $3 \mathrm{mg}$ of lyophilized tissue $/ 3 \mathrm{~mL}$ of $6 \mathrm{~N} \mathrm{HCl}$ ) and subsequently hydrolyzed in a microwave hydrolysis system (MDS-2000; CEM Co, Matthews, NC) for 45 minutes. Finally, the hydrolyzed sample was diluted with a $5 \%$ lanthanum chloride in $3 \mathrm{~N} \mathrm{HCl}$ solution. The calcium content of each test sample was determined by using 


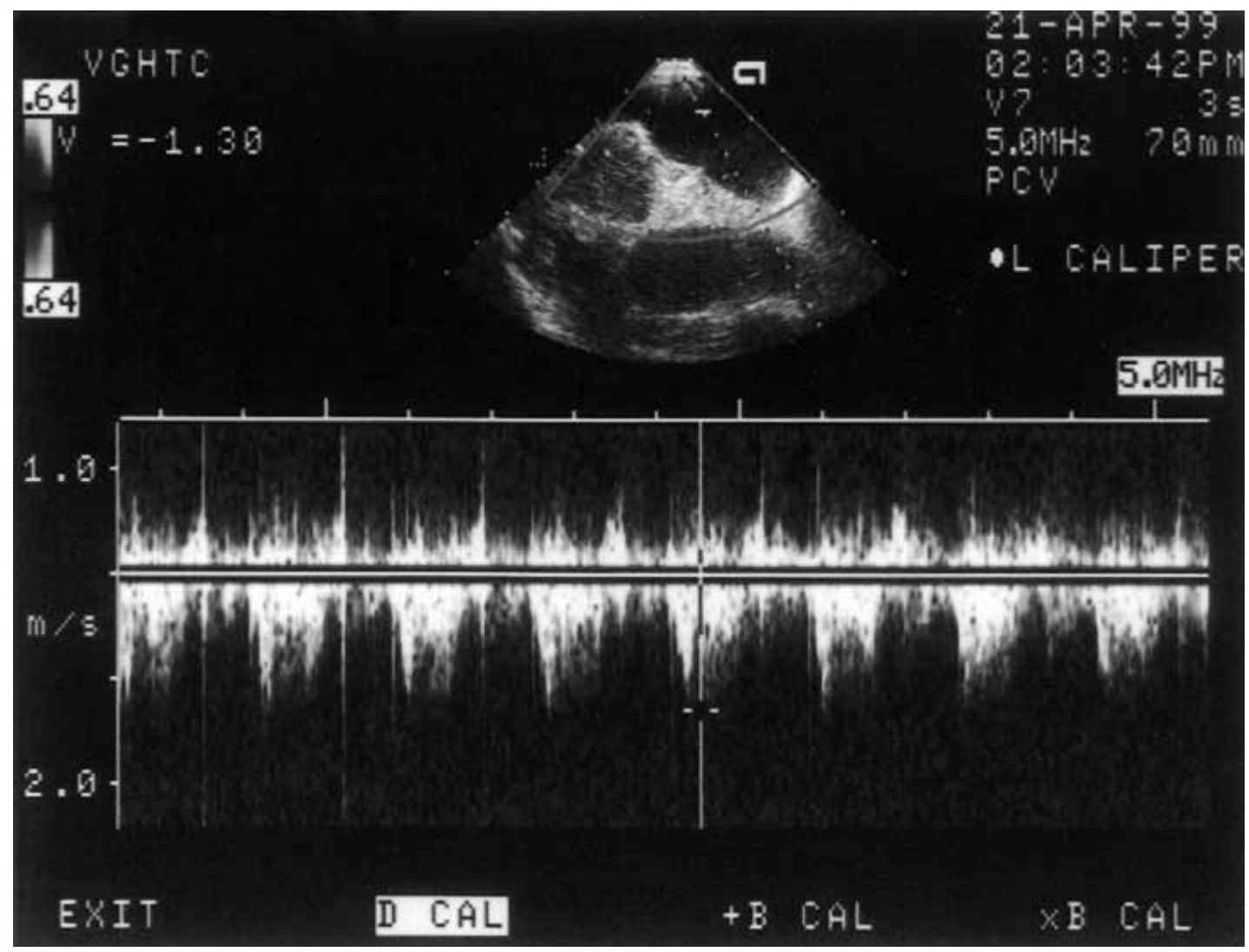

Figure 2. Continuous-wave Doppler velocity spectrum obtained in an implanted genipin-fixed conduit at 6 months postoperatively.

an atomic absorption spectrophotometer (model AA-100; PerkinElmer Inc, Norwalk, Conn) and was expressed as micrograms per milligram of dry tissue weight.

The measurements of the denaturation temperature and tensile strength of each test sample before implantation and at distinct implantation duration were used to probe the potential susceptibility of each studied group to in vivo degradation.

\section{Denaturation Temperature Measurement}

The denaturation temperatures of each studied group before implantation (taken from similarly fixed tissues that were unimplanted) and those retrieved postoperatively were measured in a Perkin-Elmer differential scanning calorimeter (model DSC-7). This technique was widely used in studying the thermal transitions of collagenous tissues. ${ }^{23}$ Details of the method used in the denaturation temperature measurement were previously described. ${ }^{24}$

\section{Tensile-Strength Measurement}

The tensile strengths of each studied group before implantation (taken from similarly fixed tissues that were unimplanted) and those retrieved at distinct implantation duration were determined by means of uniaxial measurements with an Instron material testing machine (Mini 44; Canton, Mass) at a constant speed of 10 $\mathrm{mm} / \mathrm{min}$.

\section{Statistical Analysis}

Statistical analysis for the determination of differences in the measured properties between groups was accomplished with 1-way analysis of variance and determination of CIs, which was performed with a computer statistical program (Statistical Analysis System, version 6.08; SAS Institute, Inc, Cary, NC). All data are presented as mean values \pm SD.

\section{Results}

\section{Test Valved Conduits}

In general, there was no significant difference in macroscopic observation between the genipin- and glutaraldehyde-fixed valved conduits besides the color. After fixation, it was found that the color of the genipin-fixed valved conduit became dark bluish, whereas the glutaraldehyde-fixed valved conduit turned yellowish.

\section{Echocardiographic Examination}

Echocardiography revealed that the motion of the valvular leaflets in both the glutaraldehyde- and genipin-fixed conduits was satisfactory. Figure 1 presents representative 2dimensional echocardiographic images of the valvular leaflet motion of a genipin-fixed conduit obtained at 6 months post- 


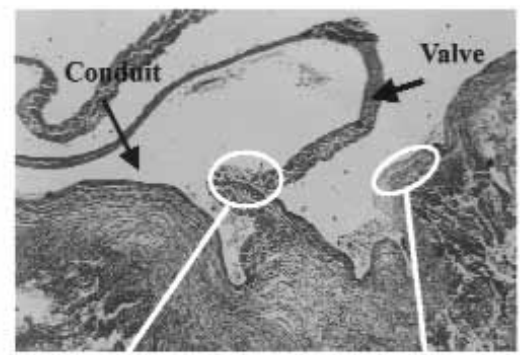

$\times 40$
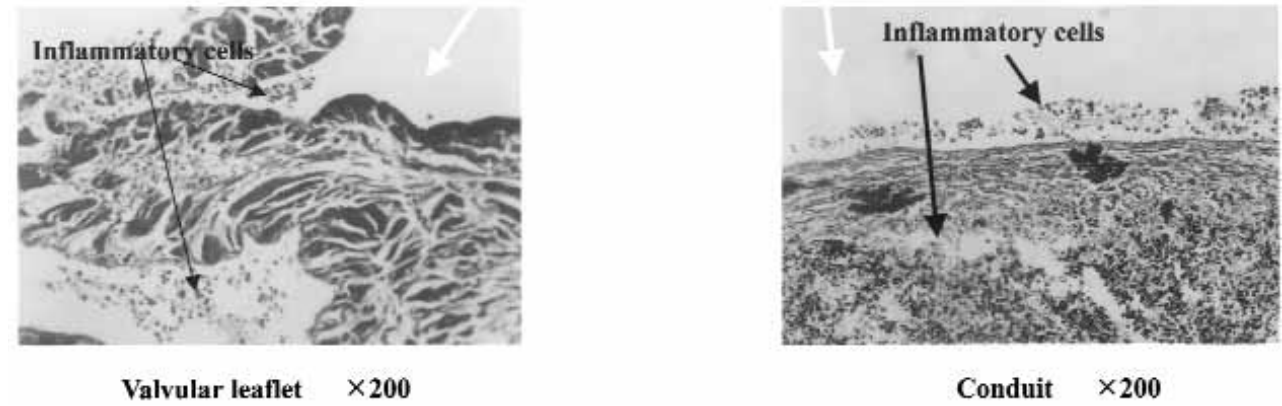

A

Valvular leaflet $\quad \times 200$

Conduit $\times \mathbf{2 0 0}$

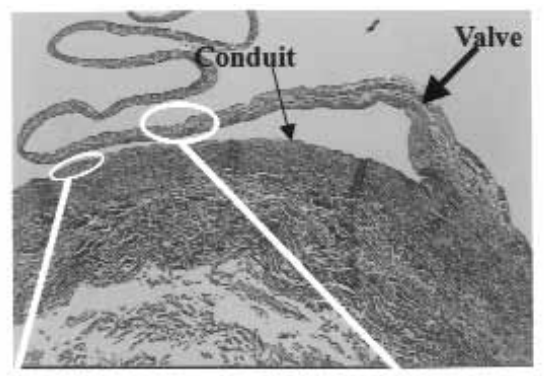

$\times 40$

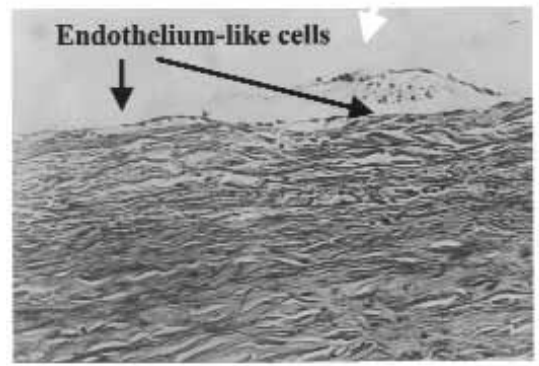

B

Conduit $\times \mathbf{2 0 0}$

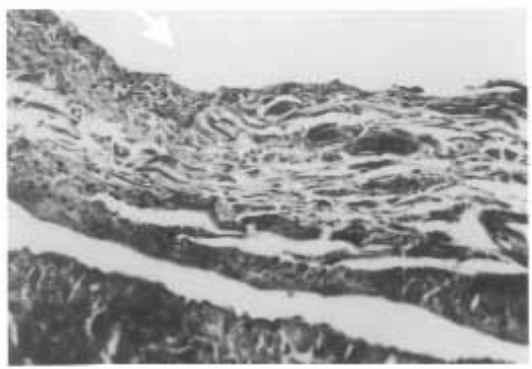

Valvular leaflet $\times \mathbf{2 0 0}$

Figure 3. Photomicrographs of longitudinal sections of the glutaraldehyde- and genipin-fixed valved conduits stained with hematoxylin and eosin and retrieved at 1 month postoperatively: A, Glutaraldehyde-fixed valved conduit; B, genipin-fixed valved conduit.

operatively. As shown, the 2 leaflets in the genipin-fixed conduit were fully open during opening (Figure 1, A) and coapted tightly at closure (Figure 1, B). An example of the continuous-wave Doppler velocity spectrum obtained in an implanted genipin-fixed conduit at 6 months postoperatively is shown in Figure 2. The peak transvalvular pressure gradient measured by using a simplified Bernoulli equation was approximately $4 \mathrm{~mm} \mathrm{Hg}$ for both the glutaraldehyde- and genipin-fixed conduits. No valvular regurgitation was observed in any of the studied cases. 


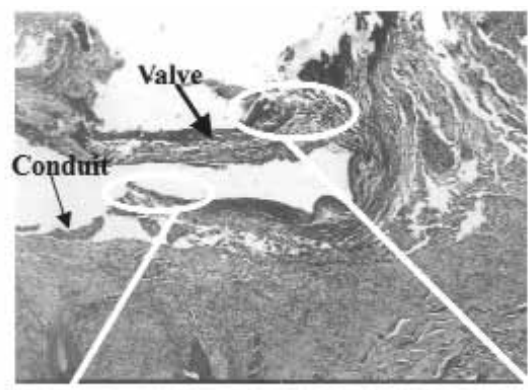

$\times 40$

A

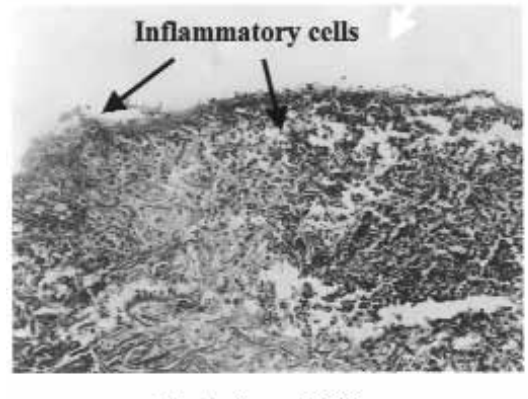

Conduit $\times 200$

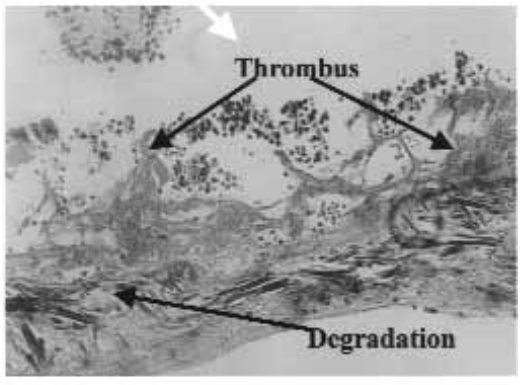

Valvular leaflet $\quad \times 200$
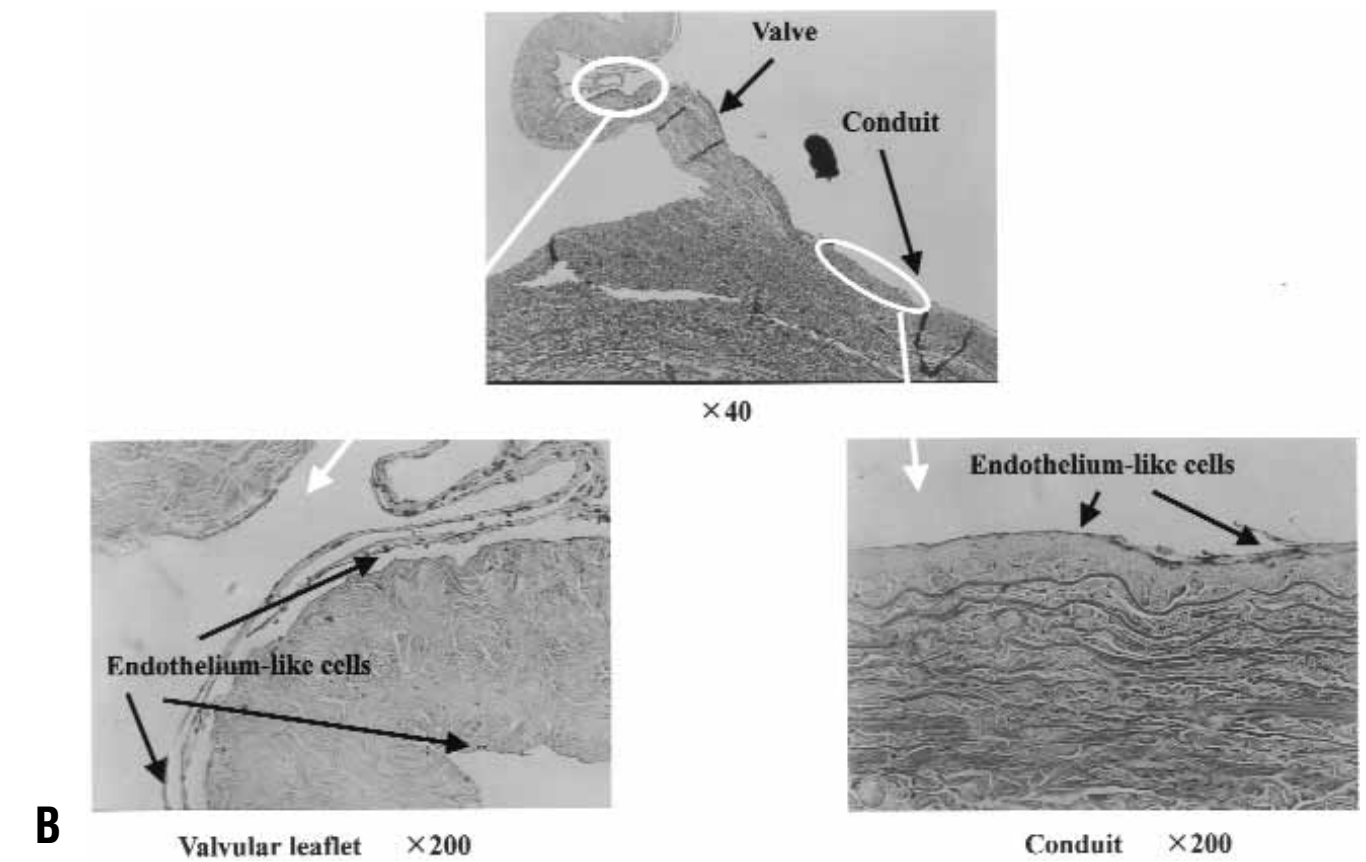

Figure 4. Photomicrographs of longitudinal sections of the glutaraldehyde- and genipin-fixed valved conduits stained with hematoxylin and eosin and retrieved at 6 months postoperatively: A, Glutaraldehyde-fixed valved conduit; B, genipin-fixed valved conduit.

\section{Histologic Findings}

At retrieval, no cardiac failure or conduit kinking or compression was observed in any of the studied cases. All conduits were patent, with the valves intact. Photomicrographs of longitudinal sections of the glutaraldehyde- and genipin-fixed valved conduits stained with hematoxylin and eosin retrieved at 1 month postoperatively are presented in Figure 3. As shown, no significant degradation was observed in the valvular leaflet and conduit for either studied group. 


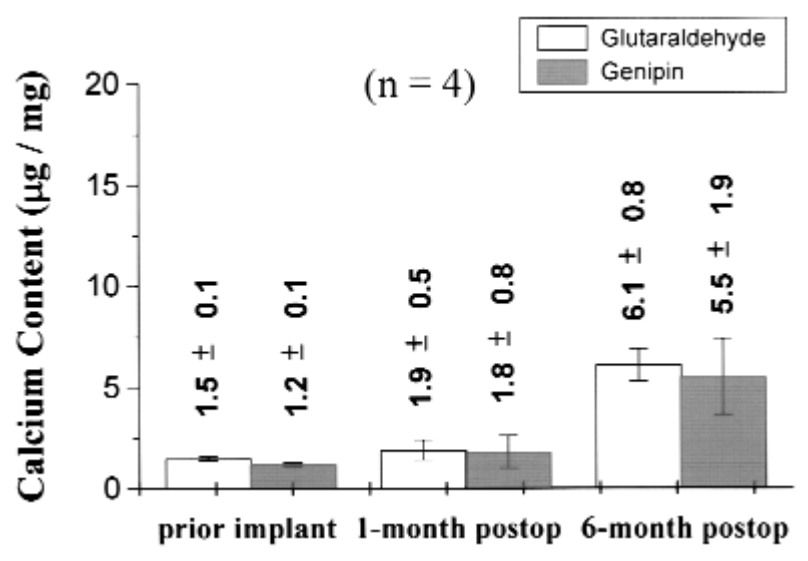

Figure 5. Calcium contents of the glutaraldehyde- and genipinfixed valvular leaflets before implantation and those retrieved at 1 and 6 months postoperatively. The calcium content of each test sample was determined by using an atomic absorption spectrophotometer and was expressed as micrograms per milligram of dry tissue weight.

Additionally, no thrombus formation was found on the surfaces of the valvular leaflet and conduit in any of the studied cases. No signs of fibrous peeling on the surfaces of the glutaraldehyde- and genipin-fixed conduits were observed.

The degrees of inflammatory reaction for the glutaraldehyde-fixed valvular leaflet and conduit appeared to be significantly more severe than those of their genipin-fixed counterparts. Of interest is that a thin layer of endotheliumlike cells formed smoothly on the surface of the genipinfixed conduit. However, there were no endothelium-like cells present on the surface of the genipin-fixed valvular leaflet at this time. In contrast, no endothelium-like cells were found, either on the surface of the valvular leaflet or on that of the conduit for the glutaraldehyde-fixed group.

At 6 months postoperatively, there was still no evidence of luminal fibrous peel in all the valved conduits studied (Figure 4). Degradation of valvular leaflet in one of the glutaraldehyde-fixed conduits was observed (Figure 4, A). In this particular case, thrombus formation was also observed on the surface of the valvular leaflet. On the other hand, no apparent degradation or thrombus formation was observed on the surfaces of the genipin-fixed valvular leaflet and conduit. The degrees of inflammatory reactions for the glutaraldehydefixed valvular leaflet and conduit observed at 6 months postoperatively were still rather severe. On the contrary, no inflammatory cells were present in the genipin-fixed valvular leaflet and conduit. Additionally, endothelium-like cells were observed on the surfaces of both the genipin-fixed valvular leaflet and conduit. In contrast, no endothelium-like cells were observed on the surfaces of the valvular leaflet and conduit for the glutaraldehyde-fixed group. No significant dilatation was present in any of the retrieved conduits.

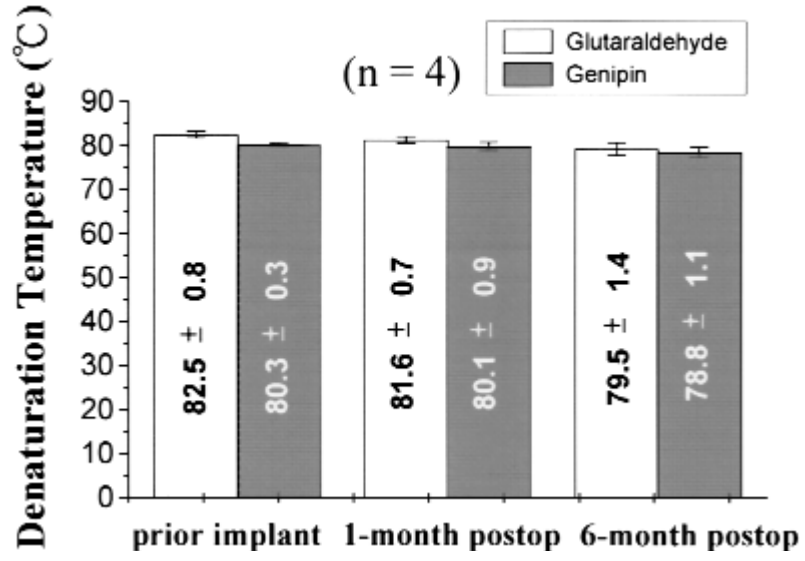

Figure 6. Denaturation temperatures of the glutaraldehyde- and genipin-fixed conduits before implantation and those retrieved at 1 and 6 months postoperatively.

\section{Calcium Content}

The calcium contents of the glutaraldehyde- and genipinfixed valvular leaflets before implantation and those retrieved at 1 and 6 months postoperatively, quantified with an atomic absorption spectrophotometer, are presented in Figure 5. As shown, the calcium contents of the samples before implantation and those retrieved at distinct implantation duration were minimal for both the glutaraldehyde- and genipin-fixed tissues. The changes in calcium content for both studied groups were not significant until 6 months postoperatively $(P<.05)$.

\section{Denaturation Temperature and Tensile Strength}

Figures 6 and 7 give the denaturation temperatures and the tensile-strength values of the glutaraldehyde- and genipinfixed conduits before implantation and those retrieved at 1 and 6 months postoperatively. Generally, the denaturation temperatures of both the glutaraldehyde- and genipin-fixed conduits declined slightly as the duration of implantation increased. However, the decline in denaturation temperature for both studied groups was not significant $(P>.05)$. Although insignificant $(P>.05)$, the tensile-strength values of the retrieved glutaraldehyde-fixed conduits appeared to be less than that of its genipin-fixed counterparts.

\section{Discussion}

Glutaraldehyde has been used extensively as a crosslinking agent for fixing biologic tissues. ${ }^{10}$ By means of its aldehyde functional groups, glutaraldehyde reacts primarily with the $\varepsilon$-amino groups of lysyl or hydroxylysyl residues within biologic tissues. The mechanism of fixation of biologic tissues with glutaraldehyde can be found in the literature. ${ }^{10}$ Polymerization of glutaraldehyde molecules in aqueous solution with observable reductions in free aldehyde have 


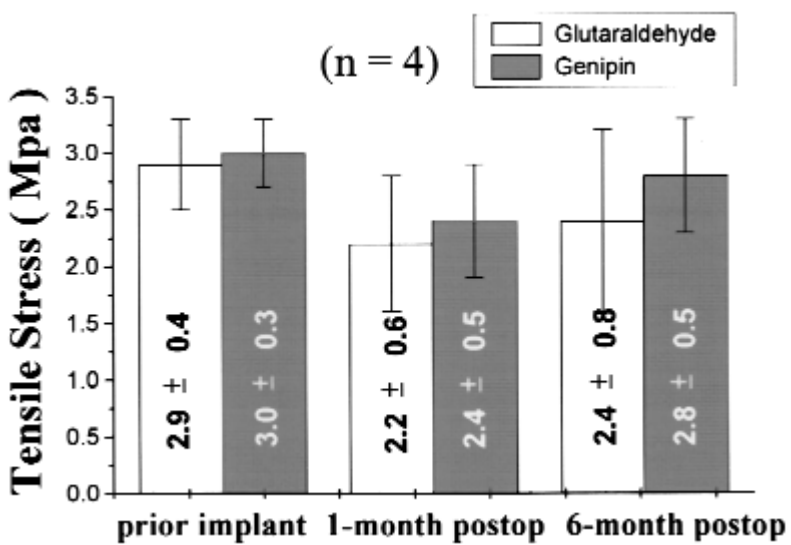

Figure 7. Tensile-strength values of the glutaraldehyde- and genipin-fixed conduits before implantation and those retrieved at 1 and 6 months postoperatively.

been reported previously. ${ }^{10,25}$ In polymerization the aldehyde functional groups of 2 glutaraldehyde molecules may undergo an aldol condensation (Figure 8). ${ }^{10}$ With glutaraldehyde polymerization, subsequent to fixation, a network crosslinking structure can be created intramolecularly and intermolecularly within collagen fibers (Figure 8).

It was found, in our previous study, ${ }^{15}$ that genipin can react with the free amino groups of lysine, hydroxylysine, or arginine residues within biologic tissues. Touyama and colleagues ${ }^{20,21}$ studied the structures of the intermediates, leading to a blue pigment produced from genipin and methylamine, the simplest primary amine. The presumed mechanism for the formation of the genipin-methylamine monomer and the blue-pigment polymers proposed by the Touyama group can be found in the literature. Briefly speaking, the genipin-methylamine monomer is formed though a nucleophilic attack by methylamine on the olefinic carbon at C-3 of genipin, followed by opening of the dihydropyran ring and attack by the secondary amino group on the resulting aldehyde group (Figure 9, A). ${ }^{20,21}$ The blue-pigment polymers are presumably formed through oxygen radical-induced polymerization and dehydrogenation of several intermediary pigments.

Similar results were also reported by Fujikawa and colleagues. ${ }^{26,27}$ In the study of the structure of a blue pigment formed from glycine and genipin, the Fujikawa group found multiple blue components. They reported that the simplest component in the blue pigment was a 1:1 adduct. From the structure of this simplest component, they proposed that genipin reacts spontaneously with an amino acid to form a nitrogen iridoid, which undergoes dehydration to form an aromatic monomer. Dimerization occurs at the second stage, perhaps by means of radical reaction. The results of the aforementioned studies ${ }^{20,21,26,27}$ suggest that genipin
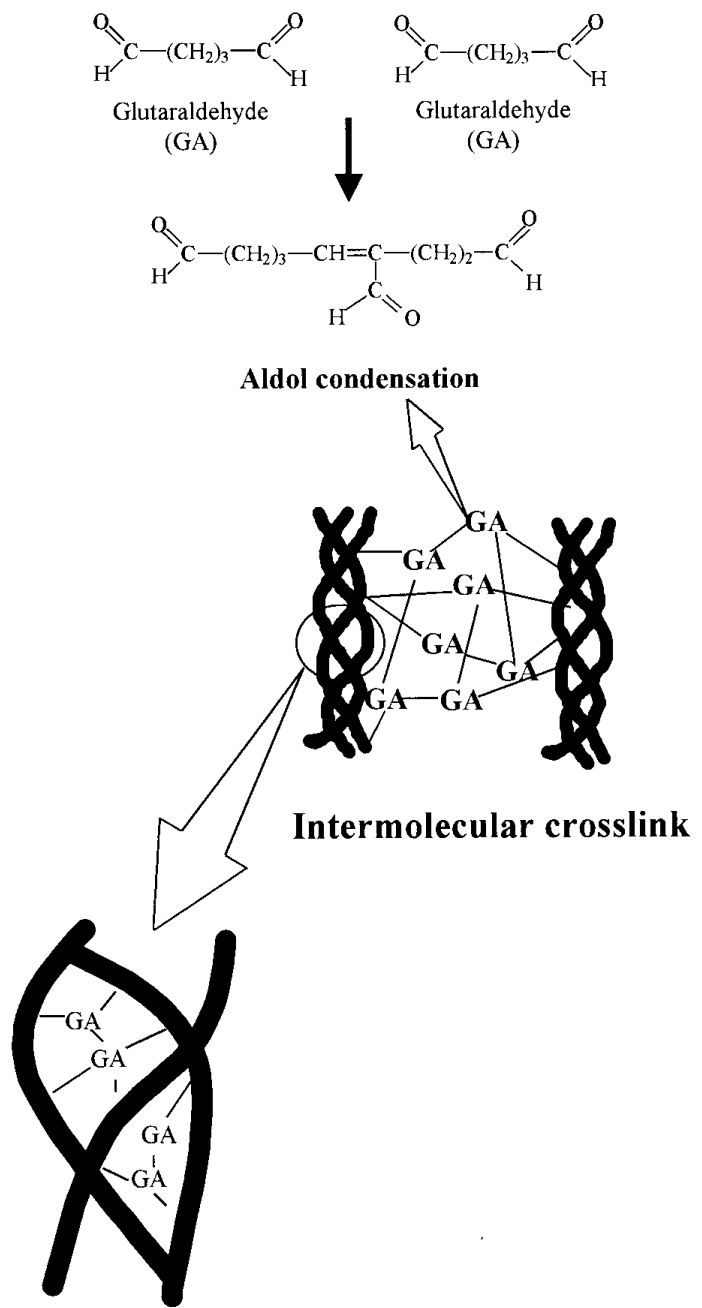

Intramolecular crosslink

Figure 8. Schematic illustration of the presumable intramolecular and intermolecular crosslinking structures of the glutaraldehydefixed tissue.

may form intramolecular and intermolecular crosslinks with cyclic structure within collagen fibers in biologic tissue (Figure 9, B).

In the study it was found that the transvalvular pressure gradients of both the glutaraldehyde- and genipin-fixed valved conduits were minimal (approximately $4 \mathrm{~mm} \mathrm{Hg}$ ) compared with those of the currently available conduits and were similar to those of the epoxy-fixed conduit reported in the studies of Noishiki, ${ }^{28}$ Sung, ${ }^{29}$ Ichikawa, ${ }^{30}$ and their colleagues. No endothelium-like cells were observed on the luminal surface of the conduit and the valvular leaflet for the glutaraldehyde-fixed group throughout the entire course of the study ( $\leq 6$ months after implantation; Figures $3, A$, and 4 , $A$ ). In contrast, endothelium-like cells were consistently observed on the luminal surface of the genipin-fixed conduit 


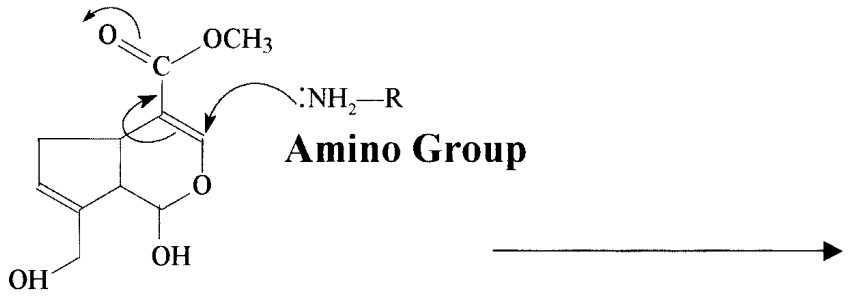

\section{Genipin (GP)}<smiles>CNC1CCC(=O)C2C(CO)=CCC12</smiles>

Intermediate

A<smiles>[R]N1C=C(C(C)=O)C2CC=C(CO)C2=C1</smiles>

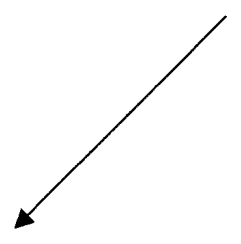
Genipin-Amino-Group Monomer

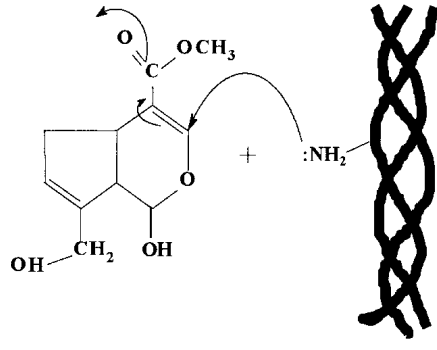

Collagen

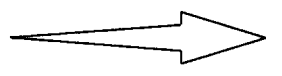

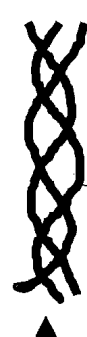

Collagen

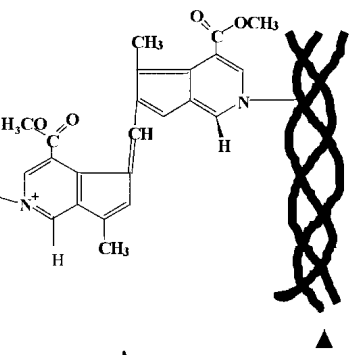

V
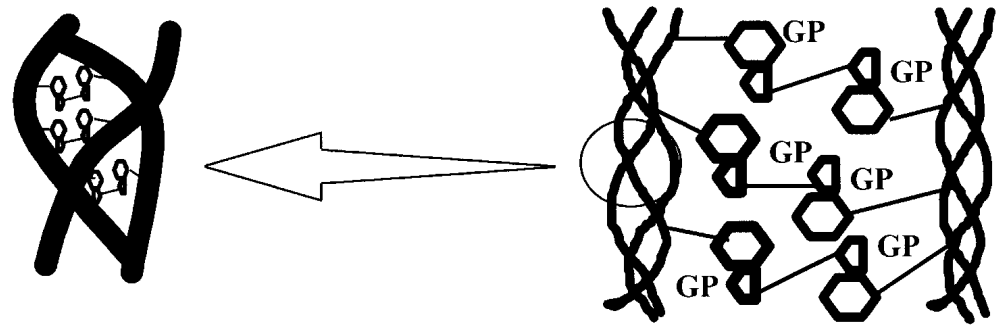

B

Intramolecular Crosslink

Intermolecular Crosslink

Figure 9. A, Presumable reaction mechanism of amino group with genipin. ${ }^{20,21}$ B, Schematic illustration of the presumable intramolecular and intermolecular crosslinking structures of the genipin-fixed tissue.

near the valvular leaflet at 1 month postoperatively (Figure $3, B)$. Moreover, endothelium-like cells were observed on the entire surface of the genipin-fixed valved conduit (both the luminal surface and the valvular leaflet) retrieved at 6 months postoperatively in all the cases studied (Figure 4, $B$ ).

Endothelialization is one of the most promising mechanisms to reduce thrombogenicity of any cardiovascular implants. ${ }^{31,32}$ It was reported that the thrombogenicity of vas- cular implants can be significantly reduced by endothelial cell lining of the luminal surface. ${ }^{33}$ In our study no thrombus formation was observed on the surfaces of the luminal graft and the valvular leaflet for the genipin-fixed group, whereas thrombus formation was observed on the glutaraldehydefixed valvular leaflet in one of the studied cases (Figure 4, A).

It was found, in an in vitro study, ${ }^{11}$ that the cytotoxicity of epoxy compound is relatively less than that of glutaralde- 
hyde. On the other hand, it was reported that genipin is significantly less cytotoxic than glutaraldehyde and epoxy compound. ${ }^{15,17}$ Host cells cannot migrate onto the cytotoxic area. Therefore, no endothelium-like cells were observed on the surfaces of the glutaraldehyde-fixed conduit and valvular leaflet (Figures 3, A, and 4, A). However, endothelial cells were found in the vicinity of the anastomoses of the epoxyfixed conduit reported on by Ichikawa and colleagues. ${ }^{30}$ In contrast, host cells (endothelium-like cells) can migrate and proliferate on the entire surface of the genipin-fixed valved conduit because of its low cytotoxicity (Figure 4,B).

Similar results were found in an in vitro cell-culture study conducted by our group. ${ }^{17}$ This indicated that the cellular compatibility of the genipin-fixed tissue was superior to its glutaraldehyde-fixed counterpart. Cytotoxicity of residual glutaraldehyde released from the glutaraldehydefixed tissue after implantation may also induce another problem, foreign-body reaction. A significantly more severe inflammatory reaction was observed for the glutaraldehydefixed conduit than its genipin-fixed counterpart throughout the entire course of the study (Figures 3, $A$ and $B$, and 4, $A$ and $B$ ).

Degeneration was observed in the glutaraldehyde-fixed leaflet retrieved at 6 months postoperatively in one of the studied cases (Figure 4,A), whereas the genipin-fixed valvular leaflets stayed intact throughout the entire course of the study. Additionally, the tensile-strength values of the retrieved glutaraldehyde-fixed conduits appeared to be slightly less than those of its genipin-fixed counterparts (Figure 7). These facts indicated that the genipin-fixed tissue may be more durable than its glutaraldehyde-fixed counterpart. As shown in Figures 8 and 9, $B$, the cyclic crosslinking structure of the genipin-fixed tissue may have a higher stereohindrance for the penetration of enzymes than the network crosslinking structure of the glutaraldehyde-fixed tissue because of the bulky heterocyclic-structure of genipin. The structure of stereohindrance may prevent enzymes from binding their corresponding substrates in biologic tissues. Therefore, the genipin-fixed tissue appeared to be more durable than its glutaraldehyde-fixed counterpart.

The calcium content of each studied group, analyzed with atomic absorption, did not change significantly until 6 months postoperatively (Figure 5). Calcification in the glutaraldehyde-fixed bioprostheses has been commonly reported clinically, ${ }^{10,13}$ but the data for the glutaraldehydefixed conduit obtained in the present study did not indicate this. Vyavahare and colleagues ${ }^{34}$ reported that ethanol pretreatment of the glutaraldehyde-fixed porcine aortic valve inhibited calcification, resulting from protein structural alterations and lipid extraction. The sterilant used in our study for each implanted sample was a graded series of ethanol solutions with a gradual increase in concentration. Therefore, the inhibition of calcification for each implanted test sample observed in our study may be due to the effect of the ethanol sterilization process.

In conclusion, although further studies are necessary, the genipin-fixed valved conduit appears to have great potential in helping mitigate the complications observed in the commercially available conduits.

\section{References}

1. Ross DN, Somerville J. Correction of pulmonary atresia with a homograft aortic valve. Lancet. 1966;2:1446-7.

2. McGoon DC, Rastelli GC, Ongley PA. An operation for the correction of truncus arteriosus. JAMA. 1968;205:59-63.

3. Rastelli GC. A new approach to "anatomic" repair of transposition of the great arteries. Mayo Clin Proc. 1969;44:1-12.

4. Kouchoukos NT, Barcia A, Bargeron LM, Kirklin JW. Surgical treatment of congenital pulmonary atresia with ventricular septal defect. $J$ Thorac Cardiovasc Surg. 1971;61:70-83.

5. Kyger ER III, Chiariello L, Hallman GL, Cooley DA. Conduit reconstruction of right ventricular outflow tract. Ann Thorac Surg. 1975; 19:277-88.

6. de Vivie ER, Hellberg K, Kutzner DD, Rahlf G, Rupprath G, Beuren AJ. Conduit repair for complex congenital heart disease with pulmonary atresia or right ventricular outflow tract obstruction. Part I: surgical results. Thorac Cardiovasc Surg. 1981;29:329-36.

7. Rupprath G, Vogt J, de Vivie ER, Beuren AJ. Conduit repair for complex congenital heart disease with pulmonary atresia or right ventricular outflow tract obstruction. Part II: early and late hemodynamic and echocardiographic findings. Thorac Cardiovasc Surg. 1981;29:33744.

8. Bull C, de Leval MR, Stark J, Taylor JFN, Macartney FJ. Use of a subpulmonary ventricular chamber in the Fontan circulation. $J$ Thorac Cardiovasc Surg. 1983;85:21-31.

9. Homann M, Haehnel JC, Mendler N, Paek SU, Holper K, Meisner H, et al. Reconstruction of the RVOT with valved biological conduits: 25 years experience with allografts and xenografts. Eur J Cardiothorac Surg. 2000;17:624-30.

10. Nimni ME, Cheung D, Strates B, Kodama M, Sheikh K. Bioprosthesis derived from cross-linked and chemically modified collagenous tissues. In: Nimni ME, editor. Collagen. Vol. III. Boca Raton (FL): CRC Press; 1988. p. 1-38.

11. Nishi C, Nakajima N, Ikada Y. In vitro evaluation of cytotoxicity of diepoxy compounds used for biomaterial modification. $J$ Biomed Mater Res. 1995;29:829-34.

12. Cipriano PR, Billingam ME, Oyer PE, Kutsche LM, Stinson EB. Calcification of porcine heart valves: a radiographic and light microscopy study. Circulation. 1982;66:1100-4.

13. Schoen FJ, Harasaki H, Kim KM, Anderson HC, Levy RJ. Biomaterial-associated calcification: pathology, mechanisms, and strategies for prevention. J Biomed Mater Res. 1988;22:11-36.

14. Speer DP, Chvapil M, Eskelson CD, Ulreich J. Biological effects of residual glutaraldehyde in glutaraldehyde-tanned collagen biomaterials. J Biomed Mater Res. 1980;14:753-64.

15. Sung HW, Huang RN, Huang LLH, Tsai CC, Chiu CT. Feasibility study of a natural crosslinking reagent for biological tissue fixation. $J$ Biomed Mater Res. 1998;42:560-7.

16. Huang LLH, Sung HW, Tsai CC, Huang DM. Biocompatibility study of a biological tissue fixed with a naturally occurring crosslinking reagent. J Biomed Mater Res. 1998;42:568-76.

17. Sung HW, Huang RN, Huang LLH, Tsai CC. In vitro evaluation of cytotoxicity of a naturally occurring crosslinking reagent for biological tissue fixation. J Biomater Sci Polym Ed. 1999;10:63-78.

18. Tsai CC, Huang RN, Sung HW, Liang HC. In vitro evaluation of the genotoxicity of a naturally occurring crosslinking agent (genipin) for biological tissue fixation. J Biomed Mater Res. 2000;52:58-65.

19. Akao T, Kobashi K, Aburada M. Enzymatic studies on the animal and intestinal bacterial metabolism of geniposide. Biol Pharm Bull. 1994; $17: 1573-6$. 
20. Touyama R, Takeda Y, Inoue K, Kawamura I, Yatsuzuka M, Ikumoto $\mathrm{T}$, et al. Studies on the blue pigments produced from genipin and methylamine. I. Structures of the brownish-red pigments, intermediates leading to the blue pigments. Chem Pharm Bull. 1994;42:668-73.

21. Touyama R, Inoue K, Takeda Y, Yatsuzuka M, Ikumoto T, Moritome $\mathrm{N}$, et al. Studies on the blue pigments produced from genipin and methylamine. II. On the formation mechanisms of brownish-red intermediates leading to the blue pigment formation. Chem Pharm Bull. 1994;42:1571-8.

22. Yoganathan AP, Cape EG, Sung HW, Williams FP, Jimoh AA. Review of hydrodynamic principles for the cardiologist: applications to the study of blood flow and jets by imaging techniques. $\mathrm{J} \mathrm{Am}$ Coll Cardiol. 1988;12:1344-50.

23. McClain PE, Wiley ER. Differential scanning calorimeter studies of the thermal transitions of collagen: implications on structure and stability. J Biol Chem. 1972;247:692-7.

24. Sung HW, Hsu CS, Lee YS, Lin DS. Crosslinking characteristics of an epoxy-fixed porcine tendon: effects of $\mathrm{pH}$, temperature, and fixative concentration. J Biomed Mater Res. 1996;31:511-8.

25. Bowes JH, Cater CW. The reaction of glutaraldehyde with proteins and other biological materials. J R Microsc Soc. 1966;85:193-200.

26. Fujikawa S, Fukui Y, Koga K. Structure of genipocyanin $\mathrm{G}_{1}$, a spontaneous reaction product between genipin and glycine. Tetrahedron Lett. 1987;28:4699-700.

27. Fujikawa S, Nakamura S, Koga K. Genipin, a new type of protein crosslinking reagent from gardenia fruits. Agric Biol Chem. 1988; 52:869-70.

28. Noishiki Y, Hata C, Tu R, Shen SH, Lin D, Sung HW, et al. Development and evaluation of a pliable biological valved conduit. Part I: preparation, biochemical properties, and histological findings. Int J Artif Organs. 1993;16:192-8.

29. Sung HW, Witzel TH, Hata C, Tu R, Shen SH, Lin D, et al. Development and evaluation of a pliable biological valved conduit. Part II: functional and hemodynamic evaluation. Int J Artif Organs. 1993;16:199-204.

30. Ichikawa Y, Noishiki Y, Kosuge T, Yamamoto K, Kondo J, Matsumoto A. Use of a bovine jugular vein graft with natural valve for right ventricular outflow tract reconstruction: a one-year animal study. J Thorac Cardiovasc Surg. 1997;114:224-33.

31. Mosquera DA, Goldman M. Endothelial cell seeding. Br J Surg. 1991;78:656-60.

32. Schneider PA, Hanson SR, Price TM, Harker LA. Confluent durable endothelialization of endarterectomized baboon aorta by early attachment of cultured endothelial cells. J Vasc Surg. 1990;11:365-72.

33. Walluscheck KP, Steinhoff G, Klein S, Haverich A. Improved endothelial cell attachment on ePTFE vascular grafts pretreated with synthetic RGD-containing peptides. Eur J Vasc Endovasc Surg. 1996;12:321-30.

34. Vyavahare N, Hirsch D, Lerner E, Baskin JZ, Schoen FJ, Bianco R, et al. Prevention of bioprosthetic heart valve calcification by ethanol preincubation: efficacy and mechanisms. Circulation. 1997;95:479-88.

\section{Availability of Journal back issues}

As a service to our subscribers, copies of back issues of The Journal of Thoracic and Cardiovascular Surgery for the preceding 5 years are maintained and are available for purchase from Mosby until inventory is depleted. Please write to Mosby, Subscription Customer Service, 6277 Sea Harbor Dr, Orlando, FL 32877, or call 800-654-2452 or 407-345-4000 for information on availability of particular issues and prices. If unavailable from the publisher, photocopies of complete issues may be purchased from Bell \& Howell Information and Learning, $300 \mathrm{~N}$ Zeeb Rd, Ann Arbor, MI 48106-1346; 734-761-4700 or 800-521-0600. 